\title{
TENSIÓN SOCIAL Y PSICOLOGÍA CULTURAL SOCIOCONSTRUCCIONISTA: PROPUESTA ANALÍTICA PARA LA CUESTIÓN CRIMINAL
}

\section{SOCIAL TENSION AND CULTURAL PSYCHOLOGY SOCIOCONSTRUCTIONIST: ANALYTICAL APPROACH FOR THE SUBJECT OF CRIMINALITY}

Irina Sibaja López ${ }^{1}$

Fecha de recepción: 21 de julio de 201 - Fecha de aceptación: 17 de noviembre de 2017

\begin{abstract}
Resumen
El presente trabajo busca reflexionar desde un enfoque teórico el tema de la criminalidad. Ello con el propósito de estudiar el aporte y la vigencia de la Teoría de la Tensión Social propuesta por Robert Merton y la Psicología Cultural Socioconstruccionista, con el fin de establecer un análisis comprensivo de la criminalidad desde su complementariedad. De ahí que se realizará, en un primer momento un recorrido por los principales postulados teóricos de ambas propuestas, mientras en un segundo momento se brindará un análisis reconociendo sus puntos de encuentro y sus divergencias. Para concluir, a pesar de ser posiciones teóricas con un origen epistemólogico diferente, tienen abordajes que resultan compatibles, ya que presentan una fuerte vinculación entre ellas, a partir de la comprensión de la cultura como centro de nuestro comportamiento en sociedad, temática que adquiere mucha relevancia en un objeto de análisis tan complejo como la criminalidad.

Palabras claves: Sociología, Tensión social, Criminalidad, Cultura, Psicología cultural.
\end{abstract}

\begin{abstract}
The document an analysis from a theoretical approach the subject of crime. This is done with the purpose of studying the contribution and validity of the Social Tension Theory proposed by Robert Merton and the Cultural Psychology Socioconstructionist, in order to establish a comprehensive analysis of criminality from its complementarity. Hence, it will be realized, at first, a tour of the main theoretical postulates of both theoretical proposals, while in the second moment an analysis will be offered, recognizing their points of encounter and their divergences. To conclude, although they are theoretical positions with a different epistemological origin, they are compatible, since they have a strong link between them, based on the understanding of culture as the center of our behavior in society, thematic that acquires a lot of relevance in an object of analysis as complex as criminality.
\end{abstract}

Keywords: Sociology, Social tension, Criminality, Culture, Cultural psychology.

1 Escuela de Sociología, Universidad de Costa Rica, irinasibaja@gmail.com 


\section{Introducción}

Uno de los retos principales de estudiar la cuestión criminal y todo lo que ello puede significar, es el tener claro las múltiples dimensiones que componen este objeto de análisis; no sólo desde el problema mismo o de la temática que nos pueda interesar; sino desde la perspectiva teórica y de los elementos epistemológicos desde los cuales partamos para su abordaje. Precisamente, antes de asumir una posición o de tomar partido al respecto del desarrollo en la teoría criminológica, resulta de esencial importancia ubicar la decisión desde un contexto general del debate, entre aquellas propuestas que podemos denominar como teorías del consenso y por otro lado, las teorías que comprendemos como las del conflicto ${ }^{2}$.

Lo anterior, en tanto se aclare que esta discusión tiene sentido con la incursión de lo que se llama criminología sociológica, ya que antes de ese quiebre conceptual se entendía la criminalidad como una patología, lo que supone la enfermedad del delincuente, configurándose desde una perspectiva médicobiológica, que en alguna medida se encuentra desde la etapa clásica liberal ilustrada a partir del siglo XVIII, y que toma más fuerza en la mitad del siglo XIX con el surgimiento del positivismo criminológico. Ello, sobre todo por estar basado en el modelo etiológico que tiene como propósito principal encontrar las causas del porqué una persona comete un delito, para que una vez detectado el delincuente se le diagnostique y se le brinde un tratamiento (la cárcel o el manicomio); constituyéndose el delito en un ente natural; planteando el tema de la patología criminal ${ }^{3}$.

Precisamente, cuando se logra ampliar la comprensión de los temas criminales desde otras perspectivas, ya no sólo la médica-biológica (unifactoriales); sino ahora desde las ciencias sociales, específicamente, la sociología y la psicología social; se incorporan un conjunto de teorizaciones con enfoques multifactoriales que marcan el inicio del desarrollo de la criminología sociológica. Dentro de esta orientación se encuentra la Escuela de Chicago (con sus tres explicaciones del comportamiento criminal más importantes), el Funcionalismo y más adelante el Estructural Funcionalismo (con solidaridad social, cohesión social y la desviación); y el Interaccionismo simbólico (o el etiquetamiento social). Así mismo, encontramos las propuestas Marxistas con un fundamento diferente a las anteriores (la lucha de clases), y en última instancia, lo que se puede llamar Criminología Crítica, que como perspectiva teórica se separa de las mencionadas al proponer una comprensión de la criminalidad, no desde la naturalidad, no desde el conflicto, sino desde una posición cuestionadora no sólo del delito, del delincuente, del sistema penal como tradicionalmente se trataba, sino desde la misma construcción de la teoría criminológica ${ }^{4}$. Dentro de esta última posición encontramos la Psicología Cultural Socioconstruccionista.

A partir de lo señalado es que tiene relevancia la distinción entre teorías del consenso y las teorías de conflicto, las tres primeras teorizaciones antes mencionadas se pueden clasificar en el consenso. Mientras que la penúltima, o sea los marxismos, y última, que si bien supone una mayor dificultad de clasificación, por su construcción teórica se pueden clasificar dentro de las del conflicto. Pavarini, añade que

2 Sobre esta tipología Ritzer indica que "teorías del consenso consideran que las normas y los valores comunes son fundamentales para la sociedad, presuponen que el orden social se basa en un acuerdo tácito y que el cambio social se produce de una manera lenta y ordenada. A diferencia de ellas, las teorías del conflicto subrayan el dominio de unos grupos sociales sobre otros, presuponen que el orden social se basa en la manipulación y el control de los grupos dominantes y que el cambio social se produce rápida y desordenadamente a medida que los grupos subordinados vencen a los grupos dominantes" (Ritzer, 1993, p.104).

3 Ver: Massimo Pavarini, Control y dominación (2002).

4 Ver los textos de Pavarini, Bergalli., Baratta, en los cuales se realiza este recorrido histórico de la criminología. 
mientras el modelo consensual postula la existencia de un consenso general en torno a los valores y a los intereses a proteger, el modelo pluralista reconoce la existencia de una multiplicidad de grupos sociales que tienen intereses diferentes y a veces en conflicto entre sí. En el modelo pluralista la ley existe no para que los individuos alcancen un acuerdo general sobre las definiciones de lo justo y de lo injusto sino exactamente para lo contrario, esto es, porque no existe ningún acuerdo general (Pavarini, 2002, p.119).

Esta diferenciación permite conocer el origen epistemológico de sus explicaciones, pero sobre todo el contexto teórico del análisis de la criminalidad que se pueda realizar a partir de cada una de ellas. Si bien, todas las teorías mencionadas tienen distinciones, saber que algunas tienen un referente común contribuye en la comprensión del objeto de estudio. No podemos decir que una explicación es mejor o peor que la otra, lo que interesa es poder analizar cuáles son los principales aportes que puede brindar cada una en la comprensión del objeto de estudio.

El presente trabajo tiene como fin reflexionar sobre la contribución de la Teoría de la Tensión Social y la Psicología Cultural Socioconstruccionista, con el fin de determinar sus puntos de encuentro y divergencias; y establecer un análisis comprensivo de la criminalidad desde su posible vinculación teórica.

Por ello, en un primer apartado se realizará un recorrido por las líneas teóricas más importantes de la cuestión criminal, para profundizar en un segundo momento sobre los principales postulados de las dos propuestas teóricas objeto del artículo, para luego brindar un análisis de sus semejanzas y diferencias, así como su uso en la comprensión de la criminalidad, en un tercer apartado.

\section{La cuestión criminal: teorías unifactoriales y multifactoriales Las teorías unifactoriales: la escuela clásica y los positivismos criminológicos}

Estas perspectivas comprenden la criminalidad como una patología, sea social o biológica, entendiendo al delincuente como una persona enferma que afecta la sociedad. Dicha posición ubica sus primeros indicios en la etapa clásica liberal ilustrada conocida como Escuela Clásica, siglo XVIII, y que se desarrolla plenamente en la mitad del siglo XIX con el Positivismo Criminológico.

Dentro de los aspectos más importantes que caracterizan la conocida Escuela Clásica se encuentran la utilización del método definido como lógico-abstracto, donde a pesar de comprender al criminal como una persona que no es sana, consideran que el delito debe dejar de ser entendido como un ente natural, para comprenderse ahora como un "ente jurídico", ligado a la construcción de la tipicidad; de ahí se regulan los distintos acontecimientos de la vida humana por medio de su creación legislativa, con el fin de garantizar su recuperación o castigo. Desligando la moral del derecho, la conciencia individual de la creación de normas colectivas ${ }^{5}$.

Aunado a lo anterior, se introduce el tema de la política penal de esta Escuela, donde se considera por primera vez la posibilidad de castigar, por medio de la idea de legitimación del poder, siendo como indica Baratta parte del desarrollo del estado liberal moderno, en el cual se da la legitimación a través de la legalidad (citado en Rivera, 2005b, p.47). Y con ello,

el «poder de castigar» se afirmará, políticamente, sobre la ideología contractual y, jurídicamente, sobre el principio de legalidad del naciente Derecho penal liberal. Así, ese poder de castigar, paulatinamente se transforma en derecho a castigar. A partir de ese momento, la historia del poder de castigar se convertirá también, en parte, en la historia del Derecho penal. Las primeras tentativas por dotar de legitimación plena a la intervención punitiva del Estado, se encuentran en las que han sido denominadas como «teorías absolutas» de la pena, o el intento por diseñar la «pena justa» (Rivera, 2005b, p.47).

$5 \quad$ Ver: Rivera, 2005b, p.46. 
Lo que es posible por medio del contrato social, y con él la configuración de las penas como mecanismos para procurar la protección de los iguales que participan del mismo, siendo el principal justificante de un nuevo orden político jurídico.

La pena se impone por causa del delito, rigiéndose por la ley del talión; todo delito supone una respuesta por razones axiológicas, ligada a la justicia; respondiendo a la pregunta de ¿por qué punir? Ubicamos aquí la teoría de la "retribución moral" propuesta por Kant donde la pena es un fin en sí mismo, además de los fundamentos de la "retribución jurídica" de Hegel quien plantea, dentro del proceso dialéctico, que la pena es la afirmación del derecho. Constituyéndose en las teorías absolutas de la pena.

Las principales contribuciones de la Escuela Clásica las podemos resumir en tres importantes aspectos. El primero es que introduce el principio de legalidad como una garantía criminal, la ley crea la ilegalidad (delito), la ley crea la pena, la ley crea el proceso y la ley crea las garantías de ejecución. Naciendo con ello, el ius puniendi estatal con un sistema penal con garantías. La segunda contribución a la comprensión de la política criminal actual, es que crea la pena privativa de libertad como una retribución al mal causado con el hecho delictivo, una pena "justa”, creando la justificación de las penas en general; todo ello desde una construcción individual del delito y de la pena. Y por último, todo su desarrollo epistemológico pone el acento en el acto, en el hecho delictivo, en aquello que colectivamente se comprende como tal y que debe ser penado por medio del Estado el cual tiene la legitimación para crearlo, producto del contrato social.

A diferencia de la anterior, aunque con algunos elementos comunes, la corriente de pensamiento de los Positivistas Criminológicos, plantea el modelo etiológico, donde lo que se buscaba era encontrar las causas del por qué se cometen los delitos, identificado el delincuente, se le diagnostique y trate por medio de la cárcel o el manicomio; planteándose el delito como un ente natural y social.

Dicha posición tiene su origen en autores como Cesare Lombroso, y fue ampliada por su discípulo Enrico Ferri, quien intentó cuestionarlo, en tanto el delito no necesariamente tiene que explicarse a partir de las características fisiológicas, sino que se deben comprender otros aspectos que lo determinan como la psicología, los factores económicos y los sociales. Sin embargo, ello no significó un cambio radical en el abordaje criminológico ya que se sigue planteando al delincuente como un enfermo. Aunado a los anteriores se suma Garófalo, quien teoriza las nociones de delito desde la idea de la patología criminal $^{6}$. Rivera afirma que

Desde el punto de vista político penal, puede afirmarse que la Escuela positiva fue una auténtica conmoción; nunca el delito y el delincuente habían sido estudiados más que como entes jurídicos.

El Positivismo tuvo la aspiración de ser un movimiento «internacional» como propio de las ciencias no sociales, sino naturales. Ahora bien, tampoco puede ser reconducido a una sola dirección. Como indica Jiménez de Asúa, dentro del Positivismo hubo claras tendencias: a) una antropológica que evaluó de modo primordial el factor endógeno (anormalidad biológica); b) otra sociológica que dio más importancia a factores o causas de la criminalidad exógenas (anormalidad social); c) otra biológico criminal que, como ciencia de síntesis, provocó el nacimiento de la ciencia de la Criminología" (Rivera, 2005b, p.55).

Por ello, se caracteriza por utilizar el método experimental, si el humano es el delincuente y el delito es resultado de una serie de factores, debe encontrar los remedios para su corrección, existe una responsabilidad colectiva. Por lo que la pena es un medio de defensa social, que se ejerce a través del orden jurídico establecido.

De ahí que se plantean las teorías relativas de la pena, preguntándose ¿para qué castigar? convirtiendo la pena en un medio para alcanzar el fin de prevenir en el futuro la comisión de delitos.

6 Ver: Massimo Pavarini, Control y dominación (2002). 
Doctrinariamente se han destacado dos grandes corrientes que la analizan, una prestando su atención al infractor (teorías de la prevención especial) y otras dando importancia a la comunidad o sociedad en su conjunto (teorías de la prevención general).

Así mismo, la prevención especial de la pena, se subdivide en prevención especial negativa y positiva. La negativa establece que la pena debe ser útil para la neutralización del transgresor, por la coerción física, tales como, aislamiento, ciertas intervenciones quirúrgicas o custodia en lugares separados, etc. Mientras que la positiva, le asigna a la pena una función terapéutica diferenciada, que busca la transformación de la conducta del transgresor; por ello la resocialización y la rehabilitación son dos términos que ubicamos dentro de esta corriente, el delincuente se trata (Rivera, 2005b).

En lo que respecta a las teorías generales de la pena, se expone que

tienen por destinatario de la pena al conjunto social, pueden presentar, dos contenidos diversos: uno, que puede denominarse como «disuasivo» (prevención general negativa). el cual se dirige a crear contra-motivaciones en los transgresores potenciales y, otro, que podría ser calificado como «expresivo» (prevención general positiva), el cual estaría orientado a declarar y reafirmar valores y reglas sociales, contribuyendo así a la integración del grupo social en torno a aquéllos (Rivera, 2005b, p.65-66).

Dentro de la prevención general negativa, Ferrajoli caracteriza dos subgrupos, las de intimidación por medio de la condena y las de intimidación por medio de la amenaza contenida en la ley (citado en Rivera, 2005b, p.67); buscando en última instancia, inhibir los impulsos delictivos de transgresores potenciales.

\section{Las teorías multifactoriales: de la criminología sociológica hasta la criminología crítica}

En los inicios del siglo XX se comienza a gestar un importante quiebre en las teorías criminológicas antes mencionadas, dejando de considerar la criminalidad como un tema biológico a una construcción social. Lo que supone que las causas del delito, son multifactoriales, donde la "realidad del delito en la que cada hecho ejecutado por uno o más autores no refleja una personalidad independiente de las condiciones ambientales, sino un individuo que lleva grabados en sí los caracteres del medio social que lo circunda" (Bergalli, Bustos y Miravalles, 1983, p.112).

La Escuela de Chicago, se ubica dentro de esta orientación, aunque si bien se sigue encontrando dentro del paradigma etiológico ya que se pregunta el porqué de la criminalidad, abandonan la explicación unicausal de la criminalidad. Con ello, establecen tres principales explicaciones teóricas sobre el tema, configurándose en teorías de alcance medio. La primera consiste en la Teoría de la Ecología Social donde realizan el análisis del orden urbano y la producción delictiva. La sociedad es entendida como una unidad ecológica, que no cumple el equilibrio en la distribución espacial urbana, donde quienes son desplazados hacia la periferia tiene más riesgos de cometer alguna conducta delictiva, ello por el

debilitamiento de los vínculos que mantenían unidos a los grupos primarios en las pequeñas comunidades a consecuencia de la vida ciudadana; modificación de las relaciones interindividuales, haciéndolas más impersonales y superficiales; pérdida del arraigo en los lugares donde se vive y relaiación de los frenos e inhibiciones en los grupos primarios bajo la influencia del ambiente urbano (Bergalli et al., 1983, p.115).

Como segundo planteamiento encontramos la Teoría de la Asociación Diferencial, donde su explicación de lo criminal, se centra en el aprendizaje social. Plantea que el delito es un fenómeno que se adquiere a partir del contacto que se tienen con las demás personas, por medio de una especie de aprendizaje de lo criminal a través del proceso de socialización, siendo diferente de una patología médico-biológica. Sutherland, su principal exponente, plantea que el comportamiento criminal siempre es aprendido (Bergalli et al., 1983). 
Mientras que la última Teoría propuesta es llamada de las Subculturas Criminales, que expone que la criminalidad se da en ámbitos sociales conflictivos. El delito es consecuencia de las organizaciones culturales diferentes, sobre las cuales no se ejerce un efectivo control social.

Junto con la Escuela de Chicago, encontramos también en este enfoque multicausal al Funcionalismo que tiene su origen principalmente en los postulados del sociólogo Emile Durkheim, que se preocupa por la temática de la solidaridad, pensada desde la cohesión social y con plena referencia en el orden moral de las sociedades. De ahí que hace un amplio desarrollo de la Teoría de la Anomía, que se da cuando "las normas y controles anteriores pierden efectividad. En tales situaciones, los seres humanos desconocen los límites que la sociedad les ha impuesto" (Bergalli et al., 1983, p.133). Siendo la sociedad un organismo vivo, donde la solidaridad y la cohesión social permiten su funcionamiento. El delito, por tanto, es una ruptura de esa vinculación social.

Reelaboraciones posteriores de este planteamiento es lo que se conoce como el Estructural Funcionalismo, y es dentro de esta orientación que encontramos a autores como Parsons y Merton. Justamente, la Teoría de la Tensión Social propuesta por este último, es objeto de análisis en el presente artículo y será desarrollada en el apartado siguiente. Podemos adelantar que en términos generales, con dicha posición se da cambio importante en su comprensión de la criminalidad, ya que se desarrolla y profundiza aún más en el concepto de anomia, convirtiéndose en la teoría del comportamiento desviado.

Así mismo, en esta orientación multicausal, encontramos el Interaccionismo Simbólico como un punto de quiebre más crítico que se comenzó a gestar desde la psicología social, y luego que retoma la sociología, desvinculándose, por primera vez, de manera plena del paradigma etiológico. Exponen que la realidad es la suma de individualidades, a partir de la cual se da un intercambio de códigos y símbolos que determinan nuestras interacciones, la realidad social se construye por medio de los contactos sociales. Quien no sigue las reglas impuestas es etiquetado, asumiendo el impacto social que esto tiene. En este punto Becker establece la Teoría del Etiquetamiento Social. Al respecto Bergalli et al. indica que

Por lo dicho, pueden afirmarse dos cosas: primero, que a partir de Becker puede hablarse ya de enfoque del etiquetamiento y, segundo, que gracias a él, el proceso mediante el cual el desviado queda individualizado como tal resulta reconocido como un proceso político en la medida en que el comportamiento así discriminado es sólo el que viola las reglas dominantes, impuestas según criterios de poder (cf. Becker, 1963, p. 163). Además, es a través de las dos etapas del labeling la constitución de la desviación, que se concreta en el momento de creación de las normas (dice Becker que «los códigos sociales crean desviación al fijar las reglas cuya infracción constituye des- viación»; 1963, p. 9) y la aplicación del sistema normativo que tiene lugar el efecto de selección, el cual termina por ser un principio unitario de ambas etapas. Asimismo, la actividad de creación y aplicación de normas da motivo al otro efecto del labelling: Ia definición del comportamiento como desviado (1983, p.151).

Otro importante punto de quiebre es el surgimiento de posiciones que buscan comprender lo criminal, desde el Marxismo, donde se dejan atrás las perspectivas del consenso, y se replantean desde la perspectiva del conflicto, partiendo de la lucha de clases como el motor de la historia. Se presentan como cuestionadores de algunos de los postulados antes indicados al discutir

la concepción tradicional de que la ley penal debe ser un instrumento de protección de la sociedad mediante la afirmación de que aquella es más bien el resultado de los intereses y los deseos de unos pocos que logran imponer su voluntad sobre la mayoría (Bergalli et al., 1983, p.141)

Siendo el elemento central de dicha disputa los intereses materiales, que orientan el desarrollo de las estructuras sociales capitalistas. El estado y el derecho desde esta posición no son neutrales, ni naturales frente a la definición de lo que se considera delito, son una expresión de la clase alta elevada a rango de ley.

Y por último, nos encontramos la llamada Criminología Crítica, que tiene una posición multicausal de lo criminal. Sin embargo, su perspectiva teórica se separa de las mencionadas, ya que cuestiona el entendimiento del tema indicando que la mirada debe estar en el delito, el delincuente, el sistema penal, 
pero también en la misma criminología, al indicar que no se pueden realizar investigaciones, sin antes estudiar ampliamente las normas y valores que definen aquello que se considera delito. Aquí encontramos la otra gran teoría que se analizará en este trabajo: la Psicología Cultural Socioconstruccionista.

En suma, luego de este recorrido nos damos cuenta que lo criminal es una temática que se ha desarrollado de manera importante en la discusión de las ciencias sociales; siendo un fenómeno que aún no se acaba de explicar y que supone un conjunto de retos y reflexiones en torno a su abordaje conceptual. De ahí el interés en el presente trabajo.

\section{Acercamiento teórico: la teoría de la tensión social y la psicología cultural socioconstruccionista}

El contexto teórico indicado en el apartado anterior es donde podemos ubicar las teorías objeto del artículo, en el cual se construyen y orientan sus principales postulados. Siendo de gran importancia como marco de referencia para su mejor comprensión.

\section{La teoría de la tensión social}

El origen de la teoría de la Tensión Social lo encontramos con el surgimiento de la perspectiva funcionalista que encuentra en las necesidades y en las demandas sociales de orden colectivo, su objeto de análisis y preocupación social. Emile Durkheim es el primer sociólogo y teórico que analiza el tema de la cohesión social, a partir de un conjunto de reglas de comportamiento, que supone valores, principios y normas comunes, que nos controlan y crean el orden deseado ${ }^{7}$, siendo un orden de carácter moral. Sin embargo, si los sujetos sociales no aprenden o internalizan los hechos sociales (esas normas) por medio de la socialización (sea primaria o secundaria), para los funcionalistas la sociedad resultaría imposible, lo que afecta la solidaridad social. De ahí que se requiera de un control efectivo de la conducta desintegradora, por lo que deben intervenir los agentes de control social. En tanto,

el aspecto que más distingue la interpretación de Durkheim del fenómeno criminal y del sistema primitivo es la radical inversión de perspectiva respecto al método positivista entonces dominante. El objeto de su análisis no es ya el hombre delincuente en su determinación biológica y social, sino más precisamente es la ruptura del mundo cultural que a nivel de los sujetos determina la violación de la norma; desde esta perspectiva la criminalidad es por primera vez interpretada en una dimensión macro sociológica (Pavarini, 2002, p.56) ${ }^{8}$.

Agrega Baratta al tema que:

Durkheim no veía ya al delincuente como "ser radicalmente antisocial, como una especie de elemento parasitario, de cuerpo extraño e inasimilable, introducido en el seno de la sociedad", sino más bien como "un agente regulador de la vida social". Esta visión general funcionalista del delito se ve acompañada en Durkheim por una teoría de los factores sociales de la anomia (2004, p.58).

Precisamente para explicar este proceso de ruptura, Durkheim acuña el concepto de anomia, como un condicionamiento colectivo que se manifiesta en el comportamiento de los individuos; mismo que sólo puede explicarse desde lo social, por ello señala que la "anomia, es malestar social, la insatisfacción por el orden social presente, que encuentra también su manifestación en el comportamiento criminal, no son otra cosa que el efecto de la naturaleza forzada de la división del trabajo" (Pavarini, 2002, p. 59).

7 Ampliar con Rivera, 2005. Recorridos y posibles formas de penalidad. 
Dentro de este contexto teórico podemos ubicar a Merton, que si bien fue discípulo de Talcott Parsons $^{9}$, se distancia de él, y retoma los elementos centrales del funcionalismo de Durkheim antes señalados, y concentra su trabajo en la conducta que no cumple con las normas previstas socialmente, en las conductas desviadas, en la anomia. Teoría que se configura en un contexto donde las demandas eran

cualitativamente diversas, de orden social y por lo tanto de política criminal que se realizaban en los mismos años en los Estados Unidos y en Europa occidental, y por ende de reflejo de las diversas situaciones socioeconómicas del Nuevo Mundo respecto de la vieja Europa. El triunfo de la sociología en la historia cultural norteamericana dependió de la capacidad mostrada por esta disciplina científica de sugerir -también desde un punto de vista teórico- las coordenadas de un sistema de control funcional a una realidad social altamente deshomogénea y conflictiva (Pavarini 2002, p.62).

Bergalli (1983) indica que podemos distanciar a Merton de Parsons, y considerar su propuesta teórica como una renovada versión, más flexible y realista del funcional estructuralismo ${ }^{10}$.

A partir de ello, Merton plantea que la forma de análisis estructural funcional más adecuada es por medio del estudio empírico, ya sea de grupos, organizaciones y culturas, a través de la representaciòn de éstos en cuestiones como "roles sociales, normas institucionales, procesos sociales, normas culturales, emociones culturalmente normadas, normas sociales, organización grupal, estructura social, mecanismos de control social, etc" (Merton, 1987, p.104). Aspectos que definen funciones estructurales de esos grupos u organizaciones, diferentes de las simples motivaciones individuales; entonces de acuerdo con Merton las funciones son las consecuencias observadas que favorecen la adaptación o ajuste de un sistema dado. De ahí que este autor desarrolla la idea de "disfunción", en tanto las estructuras pueden contribuir con el mantenimiento del sistema social o bien puede tener consecuencias negativas para él, y no obstante seguir existiendo; porque a pesar de todo resulta funcional para una parte del mismo debido a las relaciones de poder, por ejemplo la discriminación y hasta la conducta delictiva.

En lo que aquí nos interesa, el principal aporte de Merton en la comprensión de la cuestión de la criminalidad es la relación que establece entre cultura, estructura y anomia. Define la cultura como "el cuerpo organizado de valores normativos que gobiernan la conducta que es común a los individuos de determinada sociedad o grupo"; (citado en Ritzer, 1993, p.134)mientras que la estructura social es "el cuerpo organizado de relaciones sociales que mantienen entre sí diversamente los individuos de la sociedad o grupo" (Merton, 1969, citado en Ritzer, 1993, p.134). Aclara Merton que el primer concepto consiste

en objetivos, propósitos e intereses culturalmente definidos, sustentados en objetivos legítimos por todos los individuos de la sociedad, o por individuos situados en ella en una posición diferente. (...) Los objetivos predominantes implican diversos grados de sentimiento y de importancia y comprenden una estructura de referencia aspiracional. Son las cosas "por las que vale la pena esforzarse (1987, p.210).

Continúa Merton indicando que "un segundo elemento de la estructura define, regula y controla los modos admisibles de alcanzar esos objetivos. Todo grupo social acopla sus objetivos culturales a

9 Bergalli añade al respecto que "Talcott Parsons intenta en su obra precisar al máximo el inasible concepto de función con el objeto de dejar en claro que es totalmente diferente del de causalidad y que no puede confundirse con él: ((La significación del concepto de función implica concebir el sistema empírico como una "empresa en marcha". Su estructura es aquel sistema de pautas determinadas que, según lo muestra la observación empírica dentro de ciertos límites, «tienden a desarrollarse de acuerdo a una pauta constante (por ejemplo, la pauta del desarrollo de un organismo joven). La significación funcional, en este contexto, es intrínsecamente teleológica. Un proceso o conjunto de condiciones "contribuye" al mantenimiento (o desarrollo) del sistema o, al ir en detrimento de su integración, eficacia, etc., resulta disfuncional" (1983, p.36).

10 Ver este tema en Bergalli et al. 1983, p. 37 y en Ritzer, 1993, p. 128-129. 
reglas, arraigadas en las costumbres o en las instituciones, relativas a los procedimientos permisibles para avanzar hacia dichos objetivos" (1987, p.211).

Entonces, su hipótesis central, y de gran importancia para la comprensión de la cuestión criminal, es que la conducta delictiva (anómica) es un síntoma de la "disociación entre las aspiraciones culturalmente prescritas y los caminos socialmente estructurales para llegar a ellas" (Merton, 1987, p.212). A diferencia de Durkheim, menciona Bergalli que

la teoría de la anomia en la actualidad ya no se limita a describir simplemente o a establecer el fenómeno de la falta de normas. Por el contrario, se ocupa de las condiciones en que se origina dicha ausencia normativa, mejor dicho, de los procesos de cambios estructurales condicionados por la pérdida del poder de mando de dichas normas (198, p.133).

Por tanto, la sociedad nos impone un conjunto de metas culturales que debemos alcanzar en el actual sistema capitalista y de mercado (que supone éxito si consumo ciertos bienes materiales); sin embargo, a pesar de esto, el sistema no garantiza en todos los roles sociales establecidos los medios institucionalizados para alcanzar dichos objetivos, por lo que puede surgir la desviación.

De ahí que para Merton el sistema social, impone retos a los individuos y aquellos que alcanzan las metas por los medios no permitidos o institucionalizados, se convierten en individuos anómicos, en mayor o en menor grado ${ }^{11}$. En este sentido, el autor propone la Tipología de los Modos de Adaptación Individual ${ }^{12}$, ya que para él si bien la cultura cubre por igual a todos los individuos de la sociedad, con independencia de sus diferencias idiosincráticas a lo largo de sus vidas; la reacción ante la contradicción entre metas y medios, ocurre con diferente frecuencia y en grupos diferentes de la sociedad, precisamente porque los individuos que integramos los estratos estamos expuestos de manera diferente al estímulo cultural y a las restricciones sociales (Merton, 1987). Esta clasificación se caracteriza por contar con cinco tipos de adaptación que son: conformidad, innovación, ritualismo, retraimiento y rebelión ${ }^{13}$.

De ahí que Baratta nos ofrece una síntesis de lo expuesto al indicar que para Merton

la desviación como un producto de la estructura social, tan absolutamente normal como el comportamiento conforme a las reglas y valores predominantes. Esto significa que la estructura social no tiene sólo un efecto represivo, sino también y sobre todo un efecto estimulante sobre el comportamiento individual (2004, p.59).

\title{
Continúa Baratta indicando que
}

\begin{abstract}
el comportamiento criminal típico corresponde al segundo modelo, al de la innovación. Partiendo del principio según el cual el impulso hacia un comportamiento desviado se deriva de la discrepancia entre fines culturales y medios institucionales, Merton muestra el modo como los estratos sociales inferiores están sometidos, en la sociedad estadunidense analizada por él, a la máxima presión en este sentido. Como diversas indagaciones han demostrado, determinadas infracciones y determinados delitos son una reacción del todo 'normal' a una situación en que se da la acentuación cultural del éxito económico y que, no obstante, ofrece en escasa medida el acceso a los medios convencionales y legítimos del éxito (2004, p.62).
\end{abstract}

Lo anterior, con independencia de la posición que se ocupa en la estructura social, sea de estratos bajos (por ejemplo delitos contra la propiedad) o bien de estratos altos (delitos de cuello blanco) ${ }^{14}$. Para Merton la estructura social capitalista, produce una tendencia hacia la anomia y la conducta divergente;

11 Ver: Calderón, R (2012). Delito y cambio social en Costa Rica. Este es un texto que claramente ejemplifica el estado de anomia en Costa Rica, estableciendo la relación entre los delitos contra la propiedad y la desigualdad social.

12 Ver: Baratta A (2004), quien explica ampliamente el tema. Así como Bergalli (1983) que también profundiza en la tipología.

13 Ver: Merton, 1987.

14 Ver Baratta, 2004, p.69. 
donde la competencia por el éxito y el prestigio social son el objetivo principal, dándose en todo estrato social, por lo que el tipo de delito está determinado por los medios y recursos que tenga al alcance. De ahí que el proceso de socialización resulta central, siendo la familia el agente socializador primario por excelencia. En suma, nos expone Merton que

En la medida en que las funciones más generales de la estructura social es suministrar una base para la predictibilidad y la regularidad de la conducta social, se hace cada vez más limitada en su eficacia a medida que se disocian los elementos de la estructura social. En este punto extremo, la predictibilidad se reduce al mínimo y sobreviene lo que puede llamarse apropiadamente anomia o caos cultural (1987, p.239).

En suma, añade Pavarini

\begin{abstract}
Si la criminalidad es entonces un atentado a la conciencia común sobre la que se funda una sociedad integrada, en una sociedad fundada sobre la división social del trabajo, ¿qué significa el acto de quien viola la norma penal? En la nueva sociedad la criminalidad es anomia, esto es no aceptación del propio rol social, de la propia colocación en la jerarquía de las funciones. Este distinto estado subjetivo no es sino el efecto de una atenuación de las justificaciones culturales de esta sociedad. (...)El criminal es en este sentido distinto, no tanto porque ofende la conciencia común de un tejido social integrado sino porque rechaza su colocación social, pone en discusión un orden fundado sobre la diversidad, sobre la diferenciación de las oportunidades y de las gratificaciones (2002, p.59).
\end{abstract}

\title{
La psicología cultural socioconstruccionista
}

Para dar inicio a la discusión y al planteamiento de esta otra posición teórica, debemos tener claro que la psicología cultural es una rama disciplinar independiente del socioconstruccionismo como perspectiva teórica, que en nuestro caso se plantean de manera conjunta, siendo la segunda corriente la que orienta la forma de abordar y comprender los acontecimientos sociales desde dicha área de la psicología.

Cuando nos preguntamos sobre ¿qué es la psicología cultural?, ¿qué caracteriza esta área de conocimiento? Lo primero que podemos decir es que la encontramos dentro de la psicología social, existiendo coincidencia en los trabajos revisados ${ }^{15}$ de que su origen es reciente, y que podremos ubicarlo, en sentido estricto, en los años noventa, estableciendo en el ámbito psicológico la importancia de la cultura sobre la experiencia psíquica de los seres humanos, y con ello, las diferentes formas de repercusión psicológica derivadas del desarrollo de la persona en contextos sociales y culturales diversos.

Teniendo su referente en la antropología (Stigler, Sweder y Herdt) y en la psicología (Bruner y Cole $)^{16}$. Desde la última disciplina, Bruner expone, como antecedente ontológico, que "considerar el mundo como un flujo indiferente de información que es procesada por individuos, cada uno actuando a su manera,' supone perder de vista cómo se forman los individuos y cómo funcionan, por citar a Geertz, «no existe una naturaleza humana independiente de la cultura» (1991, p.28).

De ahí que la cultura se convierte en un elemento central de la configuración de las mentalidades, o de la construcción subjetiva de los seres humanos, sin que ello signifique que carece de un referente colectivo o estructural; tenemos aspectos culturales comunes por un lado, mientras que por otro está lo que percibimos individualmente, que se caracteriza por su diversidad. Bruner indica que

Dado que la psicología se encuentra tan inmersa en la cultura, debe estar organizada en torno a esos procesos de construcción y utilización del significado que conectan al hombre con la cultura.

15 Ver: García-Borés, 2000, y Serrano, 2008.

16 Ver: Serrano, 2008. 
Esto no nos conduce a un mayor grado de subjetividad en la psicología; es exactamente todo lo contrario. En virtud de nuestra participación en la cultura, el significado se hace público y compartido. Nuestra forma de vida, adaptada culturalmente, depende de significados y conceptos compartidos, y depende también de formas de discurso compartidas que sirven para negociar las diferencias de significado e interpretación (1991, p.28).

\title{
Serrano plantea entonces que,
}

la psicología cultural tratará así de comprender, explicar e interpretar el grado y la naturaleza de la correspondencia entre la "objetividad" de las categorías socioculturales, su doble función estructural y estructurante, y la "subjetividad" de las categorías psicológicas, tal y como son vividas y experimentadas por los agentes, a través de su capacidad estructuradora (2008, p.351).

En este sentido, desde la psicología cultural se considera la dicotomía sujeto-objeto, y por tal, las estructuras mentales y las estructuras sociales, se comprenden como dos planos de una misma realidad, dos dimensiones que se exponen desde una perspectiva ontológica.

\begin{abstract}
La antinomia individuo-sociedad, y los respectivos procedimientos de análisis que de ella se derivan, objetivista y subjetivista, pasan a ser considerados como dos momentos en la conceptualización del universo y la acción humana. La mirada psicocultural, y la construcción del objeto de estudio que ella implica, pasa por la delimitación de un espacio que reúna y busque integrar: de un lado, las posiciones sociales que los agentes ocupan en una estructura social determinada, y de otro, los mecanismos psicológicos (cognitivos, emocionales, motivacionales, etc.) que posibilitan que los agentes ordenen y clasifiquen el mundo, se guíen en él, y, sobre todo, ejerzan sus acciones y lleven a cabo sus prácticas (Serrano, 2008, p.351).
\end{abstract}

Este proceso dialéctico, supone la imperiosa necesidad del aprendizaje por parte de los individuos sociales del conjunto de prácticas culturales, que le determinan en el contexto en el que se ubica, y que una vez conocidas por ellos, pueden interpretar y hacer suyas; situación que se logra para la psicología cultural por medio del proceso de socialización, o específicamente enculturación (por su antecedente antropológico).

En suma, para esta rama de la psicología la cultura no es una variable externa, sino un elemento constitutivo del mundo psíquico; siendo ello el centro ontológico de su perspectiva. Lo anterior supone que su objeto de estudio es de orden simbólico, definido por un contexto social particular, un tiempo y espacio determinado.

De ahí que el espacio sociocultural es un campo de

\begin{abstract}
significaciones construidas históricamente, un espacio que necesita más de la interpretación comprensiva que la de la medición estadística. Para la psicología cultural, el actor social no solo reproduce las categorías socioculturales interiorizadas, sino que las recrea constantemente en el proceso discursivo mediante el que otorga sentido a sus acciones y, junto a ellas, a su vida entera. Vivir es, para el sujeto social, vivir interpretativamente. Y si bien es cierto que la vida es bastante más que la interpretación de la misma, sin esta última aquella carece de sentido y por tanto de las premisas necesarias para poder ser vivida, lo que implica que una psicología de las prácticas culturales no puede renunciar a incorporar en su aparato conceptual, tanto una específica noción de cultura como una concepción de ser humano en tanto que agente social y culturalmente contextualizado (Serrano, 2008, p.352).
\end{abstract}

Entonces, tenemos un sujeto constructor de sentidos, que a su vez es construido por un conjunto de significaciones que le permiten su ubicación en un contexto histórico específico. Haciendo evidente la importante influencia de la antropología en la construcción ontológica de esta rama de la psicología, ya que retoman, con lo antes dicho, la función estructurante de la cultura, y con ello, el papel central de la noción de significado, y por otro lado, la necesidad de establecer una ciencia de carácter interpretativo. Siendo un conocimiento que parte de la noción estructural de la cultura, en la cual lo subjetivo se encuentra dentro de un contexto socio-histórico que se establece en el centro de sus indagaciones y de sus interrogantes. 
A partir de lo mencionado, nos recuerda García-Borés que existen diferentes posiciones teóricas ${ }^{17}$ desde las cuales se puede abordar el análisis desde la psicología cultural

el análisis psicocultural será viable desde aquellas perspectivas teóricas cuya concepción del mundo mental esté cuanto menos participado, en mayor o menor medida, por la cultura. Por ello, en general los psicólogos culturales trabajamos desde alguna de tres tradiciones: inspirándose en la escuela cultural histórica vygotskiana; en la tradición psicodinámica, generalmente en versión freudiana o bien lacaniana; o desde el socioconstruccionismo, (...) que, en buena medida, recupera la tradición de pensamiento del Interaccionismo Simbólico (García-Borés, 2008, p.2).

Desde el presente trabajo, el interés se centra en el socioconstruccionismo, siendo una posición teórica que tiene su antecedente inmediato en el interaccionismo simbólico y en autores como Goffman (2000), Berger y Luckmann (2003); que plantean como objeto central la comprensión de los significados, más que la búsqueda de la explicación causal. "Le interesará más que el significado, el sentido de los hechos. Se enfrentará a la imagen de una producción científica nomotética, universal, que se encuentra siempre contextualizada cultural e históricamente caracterizada culturalmente" (GarciaBorés, 2000, p.16).

Los tres aspectos fundamentales que nos permiten comprender esta perspectiva son: la importancia que se le otorga a la subjetividad, como tema cuestionado por las psicologías positivistas, suponiendo el reconocimiento de "la persona como un agente activo con una actividad interpretativa de naturaleza simbólica, es decir receptora y otorgadora de significados" (García-Borés, 1993, p.94); el segundo tema central es el reconocimiento del desarrollo psicológico como una construcción social en el cual "la experiencia psicológica se constituye a partir de la interacción con los demás y, en este sentido, es un proceso social, tiene un origen social" (García-Borés, 2000, p.18); y por último se distingue por establecer que "los elementos que componen nuestra subjetividad son de naturaleza simbólica es lo mismo que decir que son de naturaleza cultural, puesto que la cultura es el mundo de las representaciones de la realidad, vigentes en un determinado contexto social, en un determinado momento histórico" (GarcíaBorés, 2000, p.19).

Siendo anti-esencialista por exponer claramente la importancia del relativismo tiempo/espacio, es anti-individualista por suponer la subjetividad como una construcción social, es anti-dualista por difuminar la separación sujeto/objeto; así como la comprensión de una realidad externa a los individuos sociales.

Lo anterior, evidencia los elementos que tienen en común con la psicología cultural, por lo que en algunos momentos establecer una distinción en sentido estricto, se configura en un reto perturbador para el investigador, lo importante aquí es que reconocen la subjetividad como una construcción social, cuya naturaleza es cultural.

Esta perspectiva teórica y disciplinar, ha contribuido en la cuestión de la definición de lo criminal, desde una posición crítica

proponiendo utilizar el delito como un indicador sociocultural, releyendo los efectos del encarcelamiento desde los procesos de enculturación, o profundizando en las consecuencias de la categoría cultural de "delincuente" como estigma fundamental. A su vez, disponemos de trabajos de investigación específicamente psicoculturales: sobre la mentalidad ciudadana en torno a la criminalidad, que muestran claramente la reproducción social del paradigma etiológico, la construcción social del delito, del delincuente y del inocente, o la autoexculpación de los ciudadanos respecto de la criminalidad; sobre la severidad social y la voluntad punitiva de los ciudadanos; o sobre las raíces psicoculturales de los procesos informales de control y censura (García-Borés, 2008, p.3).

17 Ver además García-Borés, 2000, y Serrano, 2008; quienes profundizan sobre la temática. 


\section{¿Posiciones teóricas incompatibles?: un análisis comprensivo de ambas posiciones teóricas}

En principio podríamos considerar que ambas posiciones teóricas son incompatibles, teniendo orientaciones disciplinares diferentes, una desde la sociología y la otra desde la psicología. Sin embargo, se parte de que en la vida cotidiana las personas necesitamos orientarnos en términos culturales, para interactuar con los demás; cada una de nuestras acciones supone conocer las reglas socialmente construidas. Desde un mismo entorno coexisten diferentes significados sociales que crean mundos, espacios vitales diferentes ${ }^{18}$, lo que Schutz (1974) denomina el mundo de la vida; y que configuran la realidad social.

Siendo en última instancia un mundo intersubjetivo, que creamos y nos constriñe, por medio del proceso de socialización, ya sea desde el plantemiento de la Tensión Social; y desde la enculturación desde la Psicología Cultural Socioconstruccionista; que en última instancia son dos términos que nos refiere a un mismo proceso colectivo; y que nos permiten explicar la relatividad de los significados, pero a su vez su objetivación en un contexto social determinado. Siendo este el primer punto de encuentro entre ambas posiciones teóricas. Con ello, se hace evidente la tensión entre lo individual y lo social, construimos la realidad, pero esta a su vez nos determina, y ambos posicionamientos teóricos nos resultan complementarios y de utilidad en el abordaje de la criminalidad.

En la actualidad, definir el medio ambiente en el cual desarrollamos nuestras vidas, biológicas y sociales, se hace cada vez más difícil. Desde los inicios de nuestra historia, los seres humanos hemos buscado transformar el mundo en el que habitamos. Esta transformación no ha sido solo física sino también simbólica, en la medida en que hemos atribuido significados a los diferentes eventos que suceden a nuestro alrededor. El acelerado crecimiento demográfico y productivo acarreado por el capitalismo y el desarrollo de la modernidad, han llevado a que actualmente sea cada vez más complejo el análisis de los diferentes temas, y sobre todo el de la criminalidad.

Y es aquí donde podemos ubicar la importancia que tiene la cultura como elemento configurador del comportamiento social, no como aspecto externo o como un referente de esa construcción, sino como dador de esos significados que nos orientan en el mundo y que guía nuestras acciones. Precisamente, desde Merton, es posible analizar de este orden social, la contradicción entre metas y medios que culturalmente han sido definidos; y en el mismo sentido se plantea en la Psicología Cultural Socioconstruccionista, en la cual lo subjetivo, esos significados individuales son socialmente configurados a partir de una cultura específica.

Lo anterior nos lleva a indicar que la cultura como un concepto teórico-metodológico, es el punto de encuentro desde nuestra perspectiva, entre las dos posiciones teóricas que nos ocupan en este trabajo.

Si bien tienen orígenes diferentes, y ubican la cultura desde lo colectivo por un lado, y lo individual por otro; no son más que análisis complementarios de una realidad, como lo criminal y la criminalidad, que tiene dos dimensiones, y que un análisis riguroso supondría abordar ambas caras de este fenómeno colectivo. Ello, debido a la mirada ontológica que comparten, ya que ambas perspectivas colocan a la cultura en un papel protagónico, como contexto creador del significado de las relaciones sociales; y del fenómeno de la cuestión criminal.

En este sentido, por medio de las dos teorías, podemos concluir que el delito es una construcción social, siendo una definición jurídica. De ahí dichas posiciones se pueden considerar útiles para la compresión de la realidad actual costarricense, al destacar el análisis de la desviación en Merton, y del comportamiento en el Socioconstruccionismo, destacando sus causas sociales y culturales, ubicadas en un tiempo y espacio dado, todo dentro de la realidad que nos determina. Y con ello, reconociendo que

18 El mundo vital es aquél en el que se desarrolla la vida cotidiana. En este sentido, retomamos a Alfred Schutz (1899-1959) quien plantea la existencia de un "mundo de la vida", dado por supuesto, en el cual tiene origen el "mundo de la vida diaria" (Ritzer, 2005, p.511). Para este autor, este mundo es intersubjetivo, nos es dado y nos constriñe, pero también actuamos sobre él, "el mundo es algo que debemos modificar por nuestras acciones o que las modifica" (Schutz, 1974, p.209). 
el delito es una configuración colectiva que se basa en las relaciones de poder y las contradicciones que se configuran en el contexto capitalista. Permitiéndonos ampliar el objeto de análisis "clásico" de la criminalidad, donde ya no es sólo el individuo, sino la estructura social y dentro de ella, el mismo ser humano como productor de significados. Brindo especial importancia al entorno social

puesto que es el campo intersubjetivo de la persona el espacio de donde extrae y negocia los significados sobre la realidad que configuran su experiencia psicológica. Un ser que se construye de y en lo social. Y, a su vez, lo social es construido por los humanos (García-Borés, 2000, p.18).

Sin embargo, epistemológicamente encontramos que son dos posiciones teóricas diferentes; que parten de contrucciones diversas sobre lo que entienden por conocimiento científico, y cómo éste se produce; la teoría de la Tensión Social es positivista, mientras que la Psicología Cultural Socioconstruccionista es interpretativa. Si bien parece una importante confrontación y a pesar de referirse a formas diversas de comprender la ciencia; ambas tienen conexiones mutuamente influyentes en el plano ontológico, a partir de una visión de ser humano y de contexto social común. Aquí la discusión se ubicaría en el abordaje metodológico a utilizar en el análisis de las dos dimensiones de una misma realidad, el contexto con Merton, y las experiencias individuales dentro de ese especio de significados que se estudian desde el Socioconstruccionismo.

La vinculación de ambas teorías permite la creación de conocimiento complementario, no excluyente, ya que desde la Tensión Social se analiza la construcción de metas y medios culturalmente determinados y construidos a partir de un contexto capitalista, mientras que el Socioconstruccionismo posibilita analizar ese entorno cultural que crea las subjetividades. Siendo por tanto el delito un indicador sociocultural.

\section{Conclusiones}

Los distintos posicionamientos teóricos, no son más que miradas particulares de la realidad social, sus coincidencias y diferencias, responden a la utilidad teórico-metodológica que el grupo de investigación le dé; sea ontológica o bien epistemológica.

De ahí que planteamos en última instancia que la Teoría de la Tensión Social y que la Psicología Cultural Socioconstruccionista son posiciones teóricas que resultan compatibles, a pesar de su origen epistemológico diferente; ya que tienen un fuerte vinculo a partir de la comprensión de la cultura como centro de nuestro comportamiento en sociedad. Permitiendo tener una perspectiva multidisciplinaria, desde la sociología por un lado, y la psicología por otro; si bien son construcciones que podríamos pensar diferentes, ontológicamente tienen vinculaciones que les permite acercase a un objeto de análisis como la criminalidad, a partir de sus temas comunes, desde dos miradas complementarias, más no contradictorias.

Ello sobre todo, como instrumento analítico de la cuestión criminal, ya sea para los que trabajan directamente en la administración penal como jueces y juezas, fiscales, defensores y defensoras, litigantes, o bien el cuerpo de científicas y científicos sociales, desde las múltiples disciplinas, que miran la realidad, y que somos parte, sin duda, de la construcción y de la reproducción del significado social de la criminalidad, esas ideas de las cuales participamos y damos sentido a nuestra cotidianidad.

Lo anterior, sobre todo con un tema complejo, que se compone de una innumerable cantidad de variables que intervienen. No se trata solo si tal o cuál teoría resulta la más apropiada o inapropiada, sino de comprender las dinámicas sociales e individuales que están de fondo en este fenómeno colectivo, y que desde la sociología jurídica se configura en un reto analítico. 


\section{Referencias}

Araya, Sandra. (2002). Las representaciones sociales: ejes teóricos para su discusión. Cuaderno de Ciencias Sociales, FLACSO. Recuperado de http://www.flacso.or.cr/index.php/ publicaciones-jb-br-jb-i-labor-editorial-jb-i/cuadernos/336-cuaderno-no-127

Baratta, Alexandro. (2004). Criminología crítica y crítica del derecho penal. Argentina, Buenos Aires. Siglo XXI Editores $1^{\circ}$ reimpresión. Recuperado de https://colectivociajpp.files.wordpress. com/2012/08/baratta-alessandro-criminologia-critica-y-critica-del-derecho-penal.pdf

Bergalli, Roberto, Bustos, Juan y Miravalles, Teresa. (1983). El pensamiento Criminológico I. Un análisis Crítico. Colombia, Bogotá. Editorial Temis Librería. Recuperado de http://www.pensamientopenal.com.ar/system/files/2016/06/doctrina43514.pdf

Bergalli, Roberto, Bustos Juan, González Carlos, Miravalles Teresa y De Sola Angél(1983b). El pensamiento Criminológico II. Estado y Control Social. Colombia, Bogotá. Editorial Temis Librería. Recuperado de https://issuu.com/unidadeserviciosofia/docs/el_pensamiento_criminologico_-_volu

Bruner, Jerome. (1991). Actos de significado. Madrid: Alianza Editorial. Recuperado de http://raulkoffman.com/wp-content/uploads/2012/07/Actos-de-significado.pdf

Calderón, Rodolfo.(2012). Delito y cambio social en Costa Rica. Costa Rica.Editorial FLACSO, $1^{\circ}$ edición. Recuperado de http://www.flacso.or.cr/index.php/publicaciones-jb-br-jb-i-labor-editorial-jb-i/ libros/372-delito-y-cambio-social-en-costa-rica

Durkheim, Emile. (1982). Las reglas de método sociológico. Argentina. Ediciones Orbis S.A., Hyspamerica.

Garcia-Borés Josep. (1993). La crítica sociocultural como intervención. En B.González Gabaldón y Guíl Bozal (Comps.), Psicologia Cultural (pp. 93-101). Sevilla: Eudema.

Garcia-Borés, Josep. (2000). Paisajes de la psicología cultural. Anuario de Psicología, vol. 31, no 4,925. Universitat de Barcelona. Recuperado de http://www.raco.cat/index.php/anuariopsicologia/ article/viewFile/61556/88412

Garcia-Borés, Josep. (2008). Análisis psicocultural de los procesos informales de control y censura social. Master Internacional Sistemas Penales Comparados y Problemas sociales. Universidad Nacional de Mar del Plata: Mar del Plata (Doc. policopiado).

Gergen, Kenneth. (1985). The social constructionism movement in modem psychology [El movimiento social construccionista en la psicologia moderna]. American Psychologist, 40, 266-275.

Goffman, Erwing. (2000). La presentación de la persona en la vida cotidiana. Argentina: Amorrortu Editores.

Ibáñez, Tomás. (1994). Psicología social construccionista. México: Universidad de Guadalajara.

Merton, Robert. (1987). Teoría y estructura sociales. México: F.C.E.

Pavarini, Massimo. (2002). Control y dominación: teorías criminológicas burguesas y proyecto hegemónico. Argentina, Buenos Aires. Siglo XXI Editores $1^{\circ}$ edición. Recuperado de http://www. pensamientopenal.com.ar/system/files/2014/12/doctrina39912.pdf

Peter, Berger y Lukmann, Tomas. (2003). La construcción social de la realidad. Argentina: Amorrortu Editores.

Ritzer, George. (1993). Teoría sociológica contemporánea. México. McGraw-Hill Inc. Recuperado de http://www.trabajosocial.unlp.edu.ar/uploads/docs/teoria_sociologica_contemporanea__ritzer__george.com).pdf

Ritzer, George. (2005). Teoría Sociológica Clásica. México: Mc Graw Hill Inc.

Rivera, Juan Ignacio. (2004). Mitologías y discursos sobre el castigo: Historias del presente y posibles escenarios. En: Autores, Textos y Temas. Ciencias Sociales; 40. Utopías del control y control de las utopías. Barcelona: OSPDH. Universidad de Barcelona, Anthropos Editorial.

Rivera, Juan Ignacio. (2005). Recorridos y posibles formas de penalidad. Barcelona: OSPDH. Universidad de Barcelona, Anthropos Editorial. 
Rivera, Juan Ignacio. (2005b). Política criminal y sistema penal: viejas y nuevas racionalidades. Barcelona: OSPDH. Universidad de Barcelona, Anthropos Editorial.

Rivera, Juan Ignacio. (2011). La memoria. Categoría epistemológica para el abordaje de la historia y las ciencias penales. En Revista Crítica Penal y Poder 2011, nº 1. Barcelona. Universidad de Barcelona. Observatorio del Sistema Penal y los Derechos Humanos. Recuperado de http://revistes. ub.edu/index.php/CriticaPenalPoder/issue/view/84

Schutz, Alfred. (1974). Estudios sobre teoría social II. Argentina, Buenos Aires: Amorrortu.

Serrano, Javier. (2008). Psicología Cultural. Kaulino, Adriana y Stecher, Antonio. (Eds): Cartografía de la Psicología contemporánea. Pluralismo y Modernidad. Santiago de Chile: LOM Ediciones. 